\title{
A NOTE ON UNIQUE CONTINUATION FOR SOLUTIONS OF THE $\infty$-MEAN VALUE PROPERTY
}

\author{
José G. Llorente \\ Universitat Autònoma de Barcelona, Departament de Matemàtiques \\ 08193 Bellaterra, Barcelona, Spain; jgllorente@mat.uab.cat
}

\begin{abstract}
We consider continous functions in euclidean domains that are solutions of a nonlinear mean value property related to the infinity laplacian. It is proved that such functions must satisfy a restricted unique continuation principle in the sense that if they vanish on a ball, they must vanish on the whole domain.
\end{abstract}

\section{Introduction}

It is well known that harmonic functions in a domain are characterized by the mean value property. If $u$ is harmonic in the domain $\Omega \subset \mathbf{R}^{n}$ then $u$ satisfies the mean value property in the sense that

$$
u(a)=\frac{1}{|B|} \int_{B} u
$$

for each $a \in \Omega$ and each ball $B=\bar{B}(a, r) \subset \Omega$ where, hereafter, $|\cdot|$ stands for Lebesgue measure in the corresponding euclidean space and $B(a, r), \bar{B}(a, r)$ denote, respectively, the open and closed balls of center $a$ and radius $r$. The so called inverse mean value property asks under which conditions a continuous functions satisfying the mean value property is harmonic. More precisely, suppose that for each $x \in \Omega$ there is a nonempty set $E(x) \subset(0, \operatorname{dist}(x, \partial \Omega))$ such that (1.1) holds for each $x \in \Omega$ and each $r \in E(x)$. One of the most standard versions of the inverse mean value property says for example that if $u$ is continuous in $\Omega$ and $E(x)$ contains arbitrarily small radii for all $x \in \Omega$ then $u$ is harmonic in $\Omega$. Therefore, it turns out that the assumption $\inf E(x)=0$ implies a posteriori that $E(x)$ can be extended to the interval $(0, \operatorname{dist}(x, \partial \Omega))$ for each $x$. See the survey $[N V]$ for a detailed account of different versions of the inverse mean value property. The mean value property for harmonic functions is the main ingredient of the interplay between classical potential theory, probability and Brownian motion (see [D]).

It is a major problem to understand which aspects of the linear theory can be carried out or adapted to some non-linear elliptic differential operators. For example, one may ask i) whether there is an stochastic process playing the role of brownian motion and ii) what is the interaction with mean value properties. In the case of the

doi:10.5186/aasfm.2014.3914

2010 Mathematics Subject Classification: Primary 31C05, 35B60, 31C45.

Key words: Mean value property, infinity laplacian, unique continuation.

Partially supported by grants MTM2010-16232 and SGR2009-420. 
so called $p$-laplacian and $\infty$-laplacian which are defined as follows:

$$
\begin{aligned}
\triangle_{p} u & =\operatorname{div}\left(\nabla u|\nabla u|^{p-2}\right), \quad 1<p<\infty, \\
\triangle_{\infty} u & =\sum u_{x_{i}} u_{x_{j}} u_{x_{i}, x_{j}}=H u(\nabla u) \cdot \nabla u,
\end{aligned}
$$

(here $H u$ denotes the hessian matrix of $u$ ) some progresses have been obtained recently from the point of view of tug-of-war games (see [PSSW] for the $\infty$-laplacian and [PS], [MPR] for the $p$-laplacian). In this note we will focus on the second direction, that is, the implications of mean value properties.

Let $\Omega \subset \mathbf{R}^{n}$ be a domain. Suppose that for each $x \in \Omega$ a set of radii $E(x) \subset$ $(0,+\infty)$ is given. We will say that the family $\mathcal{E}=\{E(x)\}_{x \in \Omega}$ is admissible if $\emptyset \neq$ $E(x) \subset(0, \operatorname{dist}(x, \partial \Omega))$ for each $x \in \Omega$. Suppose now that $u: \Omega \rightarrow \mathbf{R}$ and $\mathcal{E}=$ $\{E(x)\}_{x \in \Omega}$ is an admissible family. We say that $u$ satisfies the $\infty$-mean value property (denoted $\infty$-MVP ) in $\Omega$ with respect to the family $\mathcal{E}$ if $u$ is continous in $\Omega$ and for any $x \in \Omega$ and each $r \in E(x)$,

$$
u(x)=\frac{1}{2}\left(\sup _{\bar{B}(x, r)} u+\inf _{\bar{B}(x, r)} u\right) .
$$

As in classical potential theory, we say that $u$ satisfies the $\infty$-sub mean value property in $\Omega$ with respect to $\mathcal{E}$ if $u$ is upper semicontinous in $\Omega$ and for any $x \in \Omega$ and each $r \in E(x)$,

$$
u(x) \leq \frac{1}{2}\left(\sup _{\bar{B}(x, r)} u+\inf _{\bar{B}(x, r)} u\right) .
$$

Analogously, we say that $u$ satisfies a $\infty$-super mean value property in $\Omega$ if $u$ is lower semicontinous in $\Omega$ and for any $x \in \Omega$ and each $r \in E(x)$,

$$
u(x) \geq \frac{1}{2}\left(\sup _{\bar{B}(x, r)} u+\inf _{\bar{B}(x, r)} u\right) .
$$

Observe that $u$ satisfies the $\infty$-submean value property iff $-u$ satisfies the $\infty$-supermean value property and that $u$ satisfies the $\infty$-mean value property if $u$ satisfies both the sub and super mean value properties. When referring to an specific point $a \in \Omega$ and its corresponding set of radii $E(a)$, we will just say that $u$ satisfies the $\infty$-mean, submean or supermean value property at $a$. If $\Omega$ is bounded, $h>0$ and $u$ satisfies $(1.2)$ for $r=h$ in $\{x: \operatorname{dist}(x, \Omega)<h\}$ then $u$ is called a harmonious function. It turns out that solutions of the Dirichlet problem for the $\infty$-laplacian can be approximated by harmonious functions as $h \rightarrow 0$ (see [LG], [PSSW] and [MPR] for the $p$-laplacian).

One of the distinctive properties of harmonic functions is the strong unique continuation principle: if $u$ and $v$ are harmonic in a domain $\Omega \subset \mathbf{R}^{n}$ and $u=v$ on a ball $B \subset \Omega$, then $u=v$ in $\Omega$. The strong continuation property is much more delicate for nonlinear equations. In the case of the $\infty$-laplacian it is known to be false (see Example 3 in Section 4). However, as far as the author's knowledge, the question is still open for the $p$-laplacian if $1<p<\infty$ and $p \neq 2$. The main result in this note says, however, that under an appropriate condition on the family $\mathcal{E}$, solutions of the $\infty$-mean value property (1.2) satisfy a restricted unique continuation property. 
Let $\Omega \subset \mathbf{R}^{n}$ be a domain. An admissible family $\mathcal{E}=\{E(x)\}_{x \in \Omega}$ is lower semicontinous if there is a lower semicontinous function $\rho: \Omega \rightarrow(0,+\infty)$ with $0<\rho(x) \leq \operatorname{dist}(x, \partial \Omega)$ such that $E(x)=(0, \rho(x))$ for all $x \in \Omega$. Note that for any fixed $0<\lambda \leq 1$, the choice $\rho(x)=\lambda \operatorname{dist}(x, \partial \Omega)$ provides a lower semicontinous family.

The following is the main result of this note.

Theorem. Let $\Omega \subset \mathbf{R}^{n}$ be a domain and let $u$ be a solution of the $\infty$-mean value property in $\Omega$ with respect to a lower semicontinous admissible family $\mathcal{E}$. Suppose that $u$ vanishes on a nonempty open subset of $\Omega$. Then $u=0$ in $\Omega$.

\section{The Maximum Principle}

The following proposition says, in this context, that a single radius is enough to obtain a Maximum Principle.

Proposition 2.1. Let $u$ be upper semicontinous in a domain $\Omega$. Suppose that $u$ satisfies the $\infty$-sub mean value property in $\Omega$ with respect to some admissible family $\mathcal{E}$. Then:

1. If $u$ attains a global maximum in $\Omega$, then $u$ is constant in $\Omega$.

2. If $\Omega$ is bounded and $\limsup u(x) \leq M$ for each $\xi \in \partial \Omega$, then $u \leq M$ in $\Omega$.

Proof. The proof runs parallel to the corresponding one in the classical subharmonic case. Suppose that $u$ attains a global maximum value $M$ in $\Omega$ and define

$$
A=\{x \in \Omega: u(x)<M\}, \quad B=\{x \in \Omega: u(x)=M\} .
$$

Then by upper semicontinuity $A$ is open. To show that $B$ is open, choose $x \in B$ and $r \in E(x)$. Then it follows from (1.3) that $u=M$ in $B(x, r)$. This shows that $B$ is open. Since $\Omega$ is connected and $B \neq \emptyset$, we must have $\Omega=B$. To prove 2, extend $u$ to $\bar{\Omega}$ by defining $u(\xi)=\lim \sup _{x \rightarrow \xi} u(x)$ for $\xi \in \partial \Omega$. The extension is upper semicontinous in the compact set $\bar{\Omega}$ so it attains a maximum there, say $C$. If the maximum is attained in $\Omega$, then, from $1, u=C$ and therefore $C \leq M$. If the maximum is attained on $\partial \Omega$ then $C \leq M$ as well. In any case, $u \leq M$ in $\Omega$.

We get the corresponding Minimum Principle.

Proposition 2.2. Let $u$ be lower semicontinous in a domain $\Omega$. Suppose that $u$ satisfies an $\infty$-supermean value property in $\Omega$ with respect to any admissible family $\mathcal{E}$. Then:

1. If $u$ attains a global minimum in $\Omega$, then $u$ is constant in $\Omega$.

2. If $\Omega$ is bounded and $\liminf _{x \rightarrow \xi} u(x) \geq m$ for each $\xi \in \partial \Omega$, then $u \geq m$ in $\Omega$.

Suppose now that $u$ satisfies the $\infty$-MVP in $\Omega$ with respect to certain admissible family $\mathcal{E}=\{E(x)\}$ and that $G$ is a bounded, relatively compact subdomain of $\Omega$. In order to obtain the usual stronger versions of the Maximum and Minimum principles saying that the supremum and the infimum of $u$ in $G$ are actually attained at the boundary $\partial G$, it is natural to assume that if $x \in G$, then $E(x)$ contains small enough radii $r$ so that $B(x, r) \subset G$. That leads to impose that inf $E(x)=0$ for every $x \in \Omega$.

Corollary 2.3. Let $u$ satisfies a $\infty$-mean value property in a domain $\Omega$ with respect to an admissible family $\mathcal{E}=\{E(x)\}$. Suppose that $G$ is a bounded, relatively 
compact subdomain of $\Omega$ and that $\inf E(x)=0$ for each $x \in G$. Then,

$$
\sup _{G} u=\sup _{\partial G} u, \quad \inf _{G} u=\inf _{\partial G} u .
$$

Proof. Observe that, by the hypothesis, $u$ satisfies the $\infty$-MVP in $G$ with respect to some admissible family. Apply Propositions 2.1 and 2.2.

\section{Mean values and the infinity-laplacian}

The MVP introduced in section 1 has a close relation with the so-called infinitylaplacian. For smooth $u$ we define

$$
\triangle_{\infty} u=\sum_{i, j=1}^{n} u_{x_{i}} u_{x_{j}} u_{x_{i}, x_{j}}=H u(\nabla u) \cdot \nabla u,
$$

where $H u$ stands for the hessian matrix of $u$.

The following proposition is well known but we include it here for completeness.

Proposition 3.1. Let $u \in \mathcal{C}^{2}(\Omega)$. Suppose that $a \in \Omega$ and $\nabla u(a) \neq 0$. Then

$$
\lim _{r \rightarrow 0} \frac{1}{r^{2}}\left[\frac{1}{2}\left(\sup _{B(a, r)} u+\inf _{B(a, r)} u\right)-u(a)\right]=\frac{H u(a)(\nabla u(a)) \cdot \nabla u(a)}{2|\nabla u(a)|^{2}} .
$$

Proof. Fix $a \in \Omega$. Take $r>0$ such that $\bar{B}(a, r) \subset \Omega$. Then, if $h \in \mathbf{R}^{n},|h| \leq r$, we have

$$
u(a+h)=u(a)+\nabla u(a) \cdot h+\frac{1}{2} H u(a)(h) \cdot h+\epsilon(h),
$$

where the error term $\epsilon(h)$ satisfies $\frac{\epsilon(h)}{|h|^{2}} \rightarrow 0$ as $h \rightarrow 0$. Since $|h| \leq r$, we will just write $o\left(r^{2}\right)$ instead of the different error terms which will appear in successive estimates in the proof of the proposition.

Suppose that the maximum and minimum of

$$
h \rightarrow \nabla u(a) \cdot h+\frac{1}{2} H u(a)(h) \cdot h
$$

over $\bar{B}(0, r)$ are attained, respectively, at $h_{r}^{+}$and $h_{r}^{-} \in \bar{B}(0, r)$. It is easy to check that

$$
\lim _{r \rightarrow 0} \frac{h_{r}^{+}}{r}=\frac{\nabla u(a)}{|\nabla u(a)|}, \quad \lim _{r \rightarrow 0} \frac{h_{r}^{-}}{r}=-\frac{\nabla u(a)}{|\nabla u(a)|} .
$$

Indeed, note that, from the choice of $h_{r}^{+}$it follows

$$
|\nabla u(a)|+r^{2} \frac{H u(a)(\nabla u(a)) \cdot \nabla u(a)}{2|\nabla u(a)|^{2}} \leq \nabla u(a) \cdot \frac{h_{r}^{+}}{r}+\frac{r^{2}}{2} H u(a)\left(\frac{h_{r}^{+}}{r}\right) \cdot \frac{h_{r}^{+}}{r} .
$$

So, if $r_{j} \rightarrow 0$ and $\frac{h_{r_{j}}^{+}}{r_{j}} \rightarrow \xi$, where $|\xi|=1$, we have, taking limits in (3.5), that $|\nabla u(a)| \leq \nabla u(a) \cdot \xi$ which implies that $\xi=\frac{\nabla u(a)}{|\nabla u(a)|}$. This proves the first identity in (3.4), the second being analogous. 
Now, observe that

$$
\begin{aligned}
& \max _{|h| \leq r} u(a+h)=u(a)+\nabla u(a) \cdot h_{r}^{+}+\frac{1}{2} H u(a)\left(h_{r}^{+}\right) \cdot h_{r}^{+}+o\left(r^{2}\right), \\
& \min _{|h| \leq r} u(a+h) \leq u(a)-\nabla u(a) \cdot h_{r}^{+}+\frac{1}{2} H u(a)\left(h_{r}^{+}\right) \cdot h_{r}^{+}+o\left(r^{2}\right),
\end{aligned}
$$

so,

$$
\frac{1}{2}\left(\sup _{B(a, r)} u+\inf _{B(a, r)} u\right) \leq u(a)+\frac{1}{2} H u(a)\left(h_{r}^{+}\right) \cdot h_{r}^{+}+o\left(r^{2}\right) .
$$

Dealing with $h_{r}^{-}$we obtain, in an analogous way

$$
\frac{1}{2}\left(\sup _{B(a, r)} u+\inf _{B(a, r)} u\right) \geq u(a)+\frac{1}{2} H u(a)\left(h_{r}^{-}\right) \cdot h_{r}^{-}+o\left(r^{2}\right),
$$

and the conclusion follows from (3.4), (3.8) and (3.9).

The following corollary says in particular that if the sets $E(x)$ contain arbitrarily small radii then smooth solutions of the $\infty$-MVP are also solutions of the $\infty$-laplacian.

Corollary 3.2. Let $\Omega \subset \mathbf{R}^{n}$ a domain, $u \in \mathcal{C}^{2}(\Omega)$ and $a \in \Omega$. Suppose that $\inf E(a)=0$. Then:

1. If $u$ satisfies the $\infty$-sub mean value property at a with respect to $E(a)$, then $\triangle_{\infty} u(a) \geq 0$.

2. If $u$ satisfies the $\infty$-super mean value property at $a$ with respect to $E(a)$, then $\triangle_{\infty} u(a) \leq 0$.

3. If $u$ satisfies the $\infty$-mean value property at $a$ with respect to $E(a)$, then $\triangle_{\infty} u(a)=0$.

The following proposition is also elementary. Following the terminology in [CEG] we say that $\triangle_{\infty} u(a) \geq 0$ in $\Omega$ in a viscosity sense if $u$ is upper semicontinous in $\Omega$ and for every local maximum $a \in \Omega$ of $u-\phi$, where $\phi$ is $\mathcal{C}^{2}$ in some neighbourhood of $a$, it follows that $\triangle_{\infty} \phi(a) \geq 0$. Analogously, $\triangle_{\infty} u(a) \leq 0$ in $\Omega$ in viscosity sense if for every local minimum $a \in \Omega$ of $u-\phi$ where $\phi$ is $\mathcal{C}^{2}$ in some neighbourhood of $a$ it follows that $\triangle_{\infty} \phi(a) \leq 0$. Finally, $\triangle_{\infty} u(a)=0$ in $\Omega$ in viscosity sense if $\triangle_{\infty} u \geq 0$ and $\triangle_{\infty} u \leq 0$ in $\Omega$ in viscosity sense.

Proposition 3.3. Suppose that $u$ satisfies the $\infty$-submean value property in $\Omega$ with respect to the admissible family $\mathcal{E}=\{E(x)\}_{x \in \Omega}$ and that inf $E(x)=0$ for each $x \in \Omega$. Then $\triangle_{\infty} u \geq 0$ in $\Omega$ in a viscosity sense.

Proof. Let $\phi \in \mathcal{C}^{2}(\Omega)$ and suppose that $u-\phi$ has a local maximum at $a \in \Omega$. Adding up some constant we can assume that $u(a)=\phi(a)$. Then $u(x) \leq \phi(x)$ for each $x$ in some ball $B(a, r)$ and therefore

$$
\phi(a)=u(a) \leq \frac{1}{2}\left(\sup _{\bar{B}(a, r)} u+\inf _{\bar{B}(a, r)} u\right) \leq \frac{1}{2}\left(\sup _{\bar{B}(a, r)} \phi+\inf _{\bar{B}(a, r)} \phi\right)
$$

for any $r \in E(a)$, which implies the result, according to Proposition 3.1. 
Corollary 3.4. Suppose that $u$ satisfies the $\infty$-mean value property in $\Omega$ with respect to the admissible family $\mathcal{E}=\{E(x)\}_{x \in \Omega}$ and that inf $E(x)=0$ for each $x \in \Omega$. Then $\triangle_{\infty} u=0$ in $\Omega$ in a viscosity sense.

\section{Some examples and counterexamples}

The relation between the structure of the sets $E(x)$ and the regularity of the function is interesting, even for the usual mean value property (see [NV] for abundant information and references on the subject). When $n=1$, it is well known that if $I$ is an open interval of the real line, $u: I \rightarrow \mathbf{R}$ is continous and for each $x \in I$ there is a sequence $r_{k} \downarrow 0$ such that

$$
u(x)=\frac{1}{2}\left(u\left(x+r_{k}\right)+u\left(x-r_{k}\right)\right),
$$

then $u$ is linear in $I$. (See $[T]$ for Schwarz's more general version in terms of the second differences). The next proposition shows that the same is true for the $\infty$ MVP equation (1.2).

Proposition 4.1. Let $I \subset \mathbf{R}$ be an open interval and $u: I \rightarrow \mathbf{R}$ continous. Suppose that $u$ satisfies the $\infty$ - MVP with respect to some family $\mathcal{E}=\{E(x)\}$ and that inf $E(x)=0$ for each $x \in I$. Then $u$ is linear in $I$.

Proof. We first prove that $u$ is monotone in $I$. To see that, suppose on the contrary that there are $a, b \in I$ with $a<b$ and the maximum of $u$ in $[a, b]$ is strictly bigger than $\max \{u(a), u(b)\}$ (the other case is analogous). Such a maximum must be attained at $c$ with $a<c<b$. Applying the hypothesis to $c$ we get arbitrarily small radii $r$ such that

$$
u(c)=\frac{1}{2}\left(\sup _{[c-r, c+r]} u+\inf _{[c-r, c+r]} u\right) .
$$

In particular,

$$
\sup _{[c-r, c+r]} u=\inf _{[c-r, c+r]} u
$$

that is, $u$ is constant in $[c-r, c+r]$. Now an standard connectedness argument shows that $u$ must be constant in $[a, b]$, which contradicts the choice of $c$. This shows that $u$ must be monotone and therefore, for any interval $[x-r, x+r] \subset I$ we have

$$
\sup _{[x-r, x+r]} u+\inf _{[x-r, x+r]} u=u(x+r)+u(x-r)
$$

which implies that for each $x \in I, u$ satisfies (4.1) at $x$ for arbitrarily small radii $r$, so $u$ is linear in $I$.

The following construction shows that if the sets $E(x)$ are away from 0 then the $\infty$-MVP does not necessarily imply linearity.

Proposition 4.2. Let $u_{0}:[0,1] \rightarrow[0,1]$ be continous and nondecreasing, with $u_{0}(0)=0, u_{0}(1)=1$. Define $u: \mathbf{R} \rightarrow \mathbf{R}$ by setting

$$
u(x)=u_{0}(x-[x])+[x]
$$

where $[x]$ denotes the integer part of $x$. Then $u$ is continous and for each $x \in \mathbf{R}$ and each $n \in \mathbf{N}$,

$$
u(x)=\frac{1}{2}\left(\sup _{[x-n, x+n]} u+\inf _{[x-n, x+n]} u\right) .
$$


In particular, $u$ satisfies the $\infty$-mean value property in $\mathbf{R}$ with the choice $E(x)=\mathbf{N}$ for each $x \in \mathbf{R}$.

Proof. From the construction it follows that $u$ is continous and that $u(x+k)=$ $u(x)+k$ for all $k \in \mathbf{Z}$. This implies that $u$ satisfies the linear mean value property (4.1) for each $x \in \mathbf{R}$ and $r \in \mathbf{N}$. Since $u$ is monotone, this is equivalent to the $\infty$ MVP with $\mathcal{E}=\mathbf{N}$ for all $x$.

Remark 1. Observe that $u_{0}$ can be chosen such that $u_{0} \equiv 0$ in $[0, \epsilon]$ for some $\epsilon>0$. Then, even if $n=1$, the restricted unique continuation principle in the statement of the theorem does not hold if the sets of radii $E(x)$ are allowed to be away from 0 .

Remark 2. In all the examples in the rest of this section the sets $E(x)$ can be chosen of the form $(0, \operatorname{dist}(x, \Omega))$. We will just say that $u$ satisfies the $\infty$ - mean value property meaning that it is referred to admissible families of this special type.

1. If $u=\theta$ is the argument function in the plane then it is easy to check directly that $u$ satisfies the $\infty$-MVP in any domain of $\mathbf{R}^{2}$ where a continous determination of the argument is defined. If $(\rho, \theta, \varphi)$ are polar coordinates in $\mathbf{R}^{3}$ then $u=\theta$ and $u=\varphi$ satisfy the $\infty$ - MVP at $\mathbf{R}^{3} \backslash H$ where $H$ is the half-plane $\{(x, 0, z): x \leq 0, z \in \mathbf{R}\}$. A less trivial observation is that $u=\theta+\varphi$ also satisfies the $\infty$-MVP in $\mathbf{R}^{3} \backslash H$. To see that, we can assume, up to an isometry, that the point where the MVP must be checked lies on the positive $X$ axis, say $a=\left(x_{0}, 0,0\right)$ with $x_{0}>0$. Choose any $0<r<x_{0}$. Then from the symmetry of the ball $B(a, r)$ with respect to $\theta$ and $\varphi$ it follows that

$$
\sup _{B(a, r)} u+\inf _{B(a, r)} u=0=u(a)
$$

which shows that $u$ satisfies the $\infty$-MVP at $a$.

2. Canonical examples of functions satisfying the $\infty$-MVP are distance functions to linear subspaces. For instance, if $O=(0,0,0), L=\{(x, y, z): x=y=0\}$ and $H=\{(x, y, z): z=0\}$ in $\mathbf{R}^{3}$ then the functions

$$
\begin{aligned}
u(x, y, z) & =\operatorname{dist}((x, y, z), O)=\sqrt{x^{2}+y^{2}+z^{2}}, \\
v(x, y, z) & =\operatorname{dist}((x, y, z), L)=\sqrt{x^{2}+y^{2}} \\
w(x, y, z) & =\operatorname{dist}((x, y, z), H)=|z|,
\end{aligned}
$$

satisfy the $\infty$-MVP in $\mathbf{R}^{3} \backslash\{0\}, \mathbf{R}^{3} \backslash L$ and $\mathbf{R}^{3} \backslash H$, respectively. Obvious modifications can be made to consider the case of general points, lines or planes. The same examples can be constructed in any dimension.

3. Proper subsets of linear subspaces produce distance functions that are piecewise of the type in the above example. Here is a particular example: let $A=$ $\{(x, 0): x \geq 0\} \subset \mathbf{R}^{2}$ and define

$$
u(x, y)=\operatorname{dist}((x, y), A)= \begin{cases}|y|, & x \geq 0 \\ \sqrt{x^{2}+y^{2}}, & x<0\end{cases}
$$

Then $u$ satisfies the $\infty$-MVP in $\mathbf{R}^{2} \backslash A$. Note that examples of this type had been previously used to show that strong unique continuation does not hold for the equation $\triangle_{\infty} u=0([\mathrm{JLM}])$. The example above shows that strong unique continuation does 
not even hold for functions satisfying the $\infty$-MVP. Nevertheless, the theorem says that a restricted version of unique continuation does indeed hold for such functions.

4. Let $l>0$ and consider the segment $J=[-l, l] \times\{0\} \subset \mathbf{R}^{2}$. Then

$$
u(x, y)=\operatorname{dist}((x, y), J)= \begin{cases}\sqrt{(x-l)^{2}+y^{2}}, & x \geq l, \\ |y|, & |x| \leq l \\ \sqrt{(x+l)^{2}+y^{2}}, & x \leq-l\end{cases}
$$

satisfies the $\infty$-MVP in $\mathbf{R}^{2} \backslash J$.

5. We give now a necessary and sufficient condition on a closed set $F \subset \mathbf{R}^{n}$ so that the distance function to such set satisfies an $\infty$-mean value property in $\mathbf{R}^{n} \backslash F$. For $x \in \mathbf{R}^{n} \backslash F$, put $\delta(x)=\operatorname{dist}(x, F), E(x)=(0, \delta(x))$ and define the admissible family $\mathcal{E}_{F}=\{E(x)\}$.

Proposition 4.3. Let $F \subset \mathbf{R}^{n}$ be closed and define $u: \mathbf{R}^{n} \backslash F \rightarrow \mathbf{R}$ by $u(x)=$ $\delta(x)$. Then $u$ satisfies the $\infty$-mean value property in $\mathbf{R}^{n} \backslash F$ with respect to $\mathcal{E}_{F}$ if and only if $F$ verifies the following property: for each $x \in \mathbf{R}^{n} \backslash F$ there is $\xi=\xi(x) \in F$ such that

$$
B\left(x^{\prime}, 2 \delta(x)\right) \cap F=\emptyset,
$$

where $x^{\prime}=2 x-\xi(x)$. ( $x^{\prime}$ is the symmetric point of $\xi$ with respect to $x$ in the line determined by $x$ and $\xi)$.

Proof. Suppose first that $u$ satisfies the $\infty$-mean value property in $\mathbf{R}^{n} \backslash F$ with respect to $\mathcal{E}_{F}$. If the conclusion of the implication does not hold, then there is $x \in \mathbf{R}^{n} \backslash F$ such that for any $\xi \in F, B\left(x^{\prime}, 2 \delta(x)\right) \cap F \neq \emptyset$, where $x^{\prime}=2 x-\xi$. Fix such $x$. To simplify notation, we will write $\delta(x)=\delta$. Pick $\xi \in F$ such that $\delta=|x-\xi|$. From the $\infty$-MVP and continuity it follows that

$$
\sup _{B(x, \delta)} u=2 \delta
$$

Observe that $|x-\xi|=\left|x-x^{\prime}\right|=\frac{1}{2}\left|x^{\prime}-\xi\right|=\delta$. Suppose now that $a \in B\left(x^{\prime}, 2 \delta\right) \cap F$ and let $\epsilon=\frac{1}{2}\left(2 \delta-\left|a-x^{\prime}\right|\right)>0$. Choose $y \in B(x, \delta)$. We distinguish two cases. If $\left|x^{\prime}-y\right| \geq \epsilon$, apply the cosine theorem to the triangles $\xi x y$ and $x x^{\prime} y$ and get

$$
|y-\xi|^{2}=2 \delta^{2}-\left|x^{\prime}-y\right|^{2}+2|x-y|^{2}
$$

so, in particular $|y-\xi| \leq \sqrt{4 \delta^{2}-\epsilon^{2}}<2 \delta$. In the second case, if $\left|y-x^{\prime}\right|<\epsilon$, we get $|a-y| \leq\left|a-x^{\prime}\right|+\epsilon<2 \delta$. In both cases we would obtain $\sup _{B(x, \delta)} u<2 \delta$ which contradicts (4.3). This proves one implication. To prove the other implication, fix $x \in \mathbf{R}^{n} \backslash F$ and put $\delta=\delta(x), \xi=\xi(x)$. Choose $0<r<\delta$. We must see that

$$
u(x)=\delta=\frac{1}{2}\left(\sup _{\bar{B}(x, r)} u+\inf _{\bar{B}(x, r)} u\right)
$$

It is clear from the construction that the infimum in (4.4) is attained at $x+\frac{r}{\delta}(\xi-x)$ and takes the value $\delta-r$. From the hypothesis and elementary geometry if follows that the supremum must be attained at $x+\frac{r}{\delta}(\xi-x)$, with the value $\delta+r$. This proves the implication and also the proposition.

Corollary 4.4. Let $F \subset \mathbf{R}^{n}$ be closed and convex. Then $u(x)=\operatorname{dist}(x, F)$ satisfies the $\infty$-mean value property in $\mathbf{R}^{n} \backslash F$ with respect to the admissible family $\mathcal{E}_{F}$. 
Remark 3. The conclusion of Corollary 4.4 is not necessarily true inside the convex set. For instance, take $F=\left\{(x, y) \in \mathbf{R}^{2}: y \geq|x|\right\}$. If $u$ is the distance to $F$, then it is easy to check that $u$ does not satisfy $(1.2)$ at the point $(0,1)$ for any $r$ with $0<r<1 / \sqrt{2}$.

6. The results in Section 3 say that functions satisfying an $\infty$-MVP are solutions of $\triangle_{\infty}=0$ in a viscosity sense and also in a classical sense if they are $\mathcal{C}^{2}$. The following proposition shows that the converse is not necessarily true, even in the $\mathcal{C}^{2}$ case. Let $(\rho, \phi)$ denote polar coordinates in the plane. It was shown in $[\mathrm{A}]$ that $u(\rho, \phi)=\sqrt{\rho} e^{\phi / 2}$ is a solution of $\triangle_{\infty} u=0$. Note that $u$ is $\mathcal{C}^{2}$ in a neighborhood of the point $(1,0)$.

Proposition 4.5. Let $u(\rho, \phi)=\sqrt{\rho} e^{\phi / 2}$ where $(\rho, \phi)$ are polar coordinates in the plane. Then $u$ does not satisfy the $\infty$-MVP at the point $(1,0)$ for any small enough radius.

Proof. Take $r>0$ small. It is clear that the maximum and the minimum of $u$ on the closed disc $B_{r}$ centered at $(1,0)$ and radius $r$ must be attained on the circle $1+r e^{i \theta}$. Some computations show that the maximum and the minimum of the function $\theta \rightarrow u(1+r \cos \theta, r \sin \theta)$ are attained, respectively, at $\alpha_{1}$ and $\pi+\alpha_{2}$ where $0<\alpha_{2}<\alpha_{1}<\pi / 2$ and

$$
\cos \alpha_{1}=\frac{\sqrt{2-r^{2}}-r}{2}, \quad \cos \alpha_{2}=\frac{\sqrt{2-r^{2}}+2}{2} .
$$

(Note that differentiation of $u(1+r \cos \theta, r \sin \theta)$ leads to the equation $\sin \theta-\cos \theta=r$, whose roots in $[0,2 \pi]$ are precisely $\alpha_{1}$ and $\left.\pi+\alpha_{2}\right)$. Further computations give that, $1+r e^{i \alpha_{1}}=\rho_{1} e^{i \phi_{1}}$ and $1+r e^{i\left(\pi+\alpha_{2}\right)}=\rho_{2} e^{-i \phi_{2}}$ where

$$
\rho_{1}^{2}=1+r \sqrt{2-r^{2}}, \quad \rho_{2}^{2}=1-r \sqrt{2-r^{2}}
$$

and

$$
\phi_{1}=\phi_{2}=\arcsin \left(\frac{r \sqrt{2}}{2}\right)
$$

Therefore, we have

$$
\frac{1}{2}\left(\sup _{\overline{B_{r}}} u+\inf _{\overline{B_{r}}} u\right)-u(1,0)=\frac{1}{2}\left(\sqrt{\rho_{1}} e^{\phi_{1} / 2}+\sqrt{\rho_{2}} e^{-\phi_{1} / 2}\right)-1 .
$$

Let

$$
f(x)=\left(1+x \sqrt{2-x^{2}}\right)^{1 / 4} e^{\frac{1}{2} \arcsin \left(\frac{x \sqrt{2}}{2}\right)} .
$$

According to (4.6), (4.7) and (4.8) we are lead to study the sign of

$$
\frac{1}{2}(f(r)+f(-r))-1
$$

for small $r>0$. Computation of the derivatives give $f^{\prime \prime}(0)=0$ and $f^{4)}(0)=-\frac{1}{2}$ which implies that (4.9), and consequently (4.8) has negative sign for $r>0$ sufficiently small. This proves the proposition.

Remark 4. It should be pointed out that, while the Dirichlet problem for the equation $\triangle_{\infty} u=0$ has a unique solution (in the viscosity sense) in nice domains (see the survey $[\mathrm{ACJ}]$ and the references therein), this is not the case for solutions of the $\infty$-MVP, at least if the sets $E(x)$ contain arbitrarily small radii (i.e. inf $E(x)=0$ ). 
Let $u$ be the function in Proposition 4.5 and consider a small circle $D$ centered at the point $(1,0)$. We claim that the Dirichlet problem for the $\infty$-MVP equation in $D$ with boundary data $f=\left.u\right|_{\partial D}$ with respect to $\mathcal{E}$ has no solution. Indeed, if there was such a solution, say $v$, then $v$ would verify $\triangle_{\infty} v=0$ in $D$ in a viscosity sense (Corollary 3.4). Therefore we should have $u=v$ by uniqueness of viscosity solutions which is impossible because of Proposition 4.5.

\section{Proof of the theorem}

The technical property of the family $\mathcal{E}$ that will be needed in the proof is in fact weaker than lower semicontinuity. What we actually need is that for each $a \in \Omega$, each $r \in E(a)$ and any $0<t \leq 1$, there is $\eta>0$ such that if $|x-a|<\eta$ then

$$
\left\{\left(r^{2}+|x-a|^{2}-2 t r|x-a|\right)^{1 / 2}, 2|x-a|\right\} \subset E(x) .
$$

It is easy to check that if $\mathcal{E}=\{E(x)\}$ is lower semicontinuous, then it verifies (5.1). Firstly, (5.1) says that $E(x)$ must contain the radius $2|x-a|$ for $x$ close enough to $a$. On the other hand, suppose that $H$ is an hyperplane containing $a$, that $|a-\xi|=r$ and that $\xi-a$ forms with $H$ an angle $\alpha$, with $0<\cos \alpha=t \leq 1$. Let $L \subset H$ be the segment joining $a$ and the orthogonal projection of $\xi$ on $H$. Then (5.1) is also expressing the fact that if $x \in L$ is close enough to $a$ then $|x-\xi| \in E(x)$.

Let $B$ be a ball of center $a \in \mathbf{R}^{n}$. By a half ball of $B$ we will denote any of the two sets in which $B$ is divided by some hyperplane passing through $a$. The following is the key lemma of the section.

Lemma 5.1. Suppose that $u$ satisfies the $\infty-M V P$ in the domain $\Omega$ with respect to a lower semicontinuous admissible family $\mathcal{E}=\{E(x)\}$. Let $a \in \Omega, r \in E(a)$ and $B=B(a, r)$. Assume that $u=0$ in a half ball of $B$. Then $u=0$ in $B$.

Proof. We start by some reductions. First, we assume that $a=0, r=1$ so $B=B_{0}$, the unit ball. We also assume that $u=0$ in the lower unit ball $\{x=$ $\left.\left(x_{1}, \ldots, x_{n}\right) \in B_{0}: x_{n} \leq 0\right\}$ and must prove that $u=0$ in the whole unit ball. We will show that a contradiction follows from the assumption that $M=\sup _{B_{0}} u>0$.

Observe that, from equation (1.2) and the Maximum Principle applied in $\overline{B_{0}}$ (Corollary 2.3), $\inf _{B_{0}} u=-M$ and that both $M$ and $-M$ are attained at the upper unit half-sphere $S^{+}=\left\{x \in \mathbf{R}^{n}:|x|=1, x_{n} \geq 0\right\}$. Either $M$ or $-M$ must be attained at a point different from the north pole; we will assume that there is $\xi=$ $\left(\xi_{1}, \ldots, \xi_{n}\right) \in S^{+}$, with $0<\xi_{n}<1$ such that $u(\xi)=M$. Since $u=0$ at the equator of the ball, we can also assume that $\xi$ has minimal $x_{n}$-coordinate among all points $x \in S^{+}$with $u(x)=M$. Up to a rotation around the vertical axis we suppose, to simplify notation, that $0<\xi_{1}<1, \xi_{2}=\cdots=\xi_{n-1}=0$ and $0<\xi_{n}<1$, where $\xi_{1}^{2}+\xi_{n}^{2}=1$. Consider now the upper spherical half-cap $U=\left\{x \in S^{+}: x_{1} \geq \xi_{1}\right\}$.

Claim. There is $\xi^{\prime} \in U$ such that $u\left(\xi^{\prime}\right)=-M$ and $\xi_{n}^{\prime}<\xi_{n}$. From the lower semicontinuity of $\mathcal{E}$ (see also the comments at the beginning of the section) we can choose $0<\epsilon<\xi_{1}$ small enough so that $\left|a^{\prime}-\xi\right| \in E\left(a^{\prime}\right)$ where $a^{\prime}=(\epsilon, 0, \ldots, 0,0)$. Put $r^{\prime}=\left|a^{\prime}-\xi\right|$ and $B^{\prime}=\bar{B}\left(a^{\prime}, r^{\prime}\right)$. Since $\xi \in B^{\prime}, u(\xi)=M$ and $u\left(a^{\prime}\right)=0$, it follows from the MVP that the minimum value of $u$ on $B^{\prime}$ must be at most $-M$ and, from the Minimum Principle and elementary geometrical considerations, it must be attained at some $\xi^{\epsilon} \in \partial B^{\prime} \backslash B_{0}$ with $0<\xi_{n}^{\epsilon}<\xi_{n}$. Letting $\epsilon \rightarrow 0$ and taking some 
subsequence we deduce that there is $\xi^{\prime} \in U$ such that $u\left(\xi^{\prime}\right)=-M$. Since $\xi$ is the only point in $U$ with maximal $x_{n}$-coordinate we also have that $0<\xi_{n}^{\prime}<\xi_{n}$ and the claim follows.

Now we repeat the same argument with $\xi^{\prime}$ instead of $\xi$ and $-M$ instead of $M$. We conclude as above that there exists $\xi^{\prime \prime} \in S^{+}$such that $u\left(\xi^{\prime \prime}\right)=M$ and $0<\xi_{n}^{\prime \prime}<\xi_{n}^{\prime}<\xi_{n}$. The contradiction follows from the fact that we chose $\xi$ with the property that has minimal $x_{n}$-coordinate among all points $x \in S^{+}$with $u(x)=M$. This contradiction finishes the proof of the lemma.

Proof of the theorem. Let $G$ be the union of all open balls $B$ contained in $\Omega$ and such that $u=0$ on $B$. Then $G \subset \Omega$ is open and non-empty. We must see that $G=\Omega$. If not, we can choose $a \in G$ and $b \in \partial G \cap \Omega$ such that $|a-b|=\operatorname{dist}(a, \partial G)=R>0$. In particular $B(a, R) \subset G$. From the lower semicontinuity of $\mathcal{E}$ we can pick $x$ in the segment joining $a$ and $b$ so that $|x-b|<\frac{R}{2}$ and $2|x-b| \in E(x)$. It follows that the half ball $B(x, 2|x-b|) \cap\{y:(y-x) \cdot(a-x) \geq 0\}$ is contained in $B(a, R) \subset G$. Now Lemma 5.1 would imply that the whole ball $B(x, 2|x-b|)$ is contained in $G$ which contradicts that $b \in \partial G$. This finishes the proof of the theorem.

\section{References}

[A] Aronsson, G.: On certain singular solutions of the partial differential equation $u_{x}^{2} u_{x x}+$ $2 u_{x} u_{y} u_{x y}+u_{y}^{2} u_{y y}=0$. - Manuscripta Math. 47:1-3, 1984, 133-151.

[ACJ] Aronsson, G., M. G. Crandall, and P. Juutinen: A tour of the theory of absolutely minimizing functions. - Bull. Amer. Math. Soc. (N.S.) 41:4, 2004, 439-505.

[CEG] Crandall, M. G., L. C. Evans, and R. F. Gariepy: Optimal Lipschitz extensions and the infinity Laplacian. - Calc. Var. Partial Differential Equations 13:2, 2001, 123-139.

[D] Durrett, R.: Brownian motion and martingales in analysis. - Wadsworth Mathematics Series, 1984.

[LG] Le Gruyer, E.: On absolutely minimizing lipschitz extension and PDE $\triangle \infty(u)=0$. Nonlinear Differential Equations and Applications 14:1-2, 2007, 29-55.

[JlM] Juutinen, P., P. Lindqvist, and J. J. Manfredi: The infinity laplacian: examples and observations. - In: Papers on Anaysis: a volume dedicated to Olli Martio on the occasion of his 60th birthday, Report Univ. Jyväskylä 83, 2001, 207-217.

[MPR] Manfredi, J. J., M. Parvianen, and J. D. Rossi: On the definition and properties of p-harmonious functions. - Ann. Sc. Norm. Super. Pisa Cl. Sc. 11:5, 2013, 215-241.

[NV] Netuka, I., and J. VeselÝ: Mean value properties and harmonic functions. - In: Classical and modern potential theory and applications, NATO Adv. Sci. Inst. Ser. C Math. Phys. Sci. 430, Kluwer, 1994, 359-398.

[PS] Peres, Y., and S. Sheffield: Tug-of-war with noise: a game-theoretic view of the $p$ Laplacian. - Duke Math. J. 145:1, 2008, 91-120.

[PSSW] Peres, Y., O. Schramm, S. Sheffield, and D. B. Wilson: Tug-of-war and the infinity laplacian. - J. Amer. Math. Soc. 22:1, 2009, 167-210.

[T] Thomson, B.S.: Symmetric properties of real functions. - Marcel Dekker, 1994.

Received 7 June 2013 • Accepted 26 August 2013 